\title{
A 2.5D finite element and boundary element model for the ground vibration from trains in tunnels and validation using measurement data
}

\author{
Qiyun Jin*, David J Thompson, Daniel E J Lurcock, Martin G R Toward, Evangelos Ntotsios \\ Institute of Sound and Vibration Research, University of Southampton, Southampton SO17 1BJ, UK \\ *: corresponding author, email: Qiyun.Jin@soton.ac.uk
}

\begin{abstract}
A numerical model is presented for the ground-borne vibration produced by trains running in tunnels. The model makes use of the assumption that the geometry and material properties are invariant in the axial direction. It is based on the so-called two-and-a-half dimensional (2.5D) coupled Finite Element and Boundary Element methodology, in which a two-dimensional cross-section is discretised into finite elements and boundary elements and the third dimension is represented by a Fourier transform over wavenumbers. The model is applied to a particular case of a metro line built with a cast-iron tunnel lining. An equivalent continuous model of the tunnel is developed to allow it to be readily implemented in the 2.5D framework. The tunnel structure and the track are modelled using solid and beam finite elements while the ground is modelled using boundary elements. The 2.5D track-tunnel-ground model is coupled with a train consisting of several vehicles, which are represented by multi-body models. The response caused by the passage of a train is calculated as the sum of the dynamic component, excited by the combined rail and wheel roughness, and the quasi-static component, induced by the constant moving axle loads. Field measurements have been carried out to provide experimental validation of the model. These include measurements of the vibration of the rail, the tunnel invert and the tunnel wall. In addition, simultaneous measurements were made on the ground surface above the tunnel. Rail roughness and track characterisation measurements were also made. The prediction results are compared with measured vibration obtained during train passages, with good agreement.
\end{abstract}

Keywords: ground vibration; railway vibration; tunnels; wavenumber finite element; waveguide boundary element; validation measurements 


\section{Introduction}

Over the last few decades there has been a considerable expansion of underground metro lines in cities around the world. While these have many advantages in providing improved transportation in urban situations, underground trains running in tunnels produce ground-borne vibration which is transmitted into nearby buildings. This may be felt as whole-body vibration or, more commonly for tunnels, heard as ground-borne noise; in both cases this may be disturbing for the occupants of the buildings [1,2]. This is an important environmental issue both for existing metros and for new lines planned in built-up areas. With the increase in living standards and growing popularity of light-weight building structures, developers are faced with more stringent vibration and noise limits. Therefore, prediction methods are required that can provide reliable estimates of the vibration and noise from new railway lines or in new buildings due to existing lines. However, due to the uncertainty of the soil conditions and other parameters, a safety factor of as much as $10 \mathrm{~dB}$ is often considered in predictions of ground vibration or ground-borne noise $[3,4]$. The prediction models used include those based on empirical, analytical and numerical methods.

An empirical approach is often used which can avoid the need for detailed input data or complex theoretical models, but this depends on obtaining suitable measurement data appropriate to the situation considered. The American Federal Transit Authority describes a procedure that uses measured transfer functions and source terms (expressed as force densities) which are combined to predict the vibration for a new situation [5]. The source terms can be obtained from a different, although similar railway if the one considered is yet to be built, but the transfer mobilities should be measured at the location in question. Verbraken et al. [6] reviewed this procedure using theoretical modelling and concluded that the main assumptions are justified. Empirical prediction models can also be established using databases of field measurements. For example, in [7] an empirical methodology was proposed based on the analysis of over 3000 measurements for the assessment and calculation of perceptible vibration and ground-borne noise from the operation of trains in railway tunnels. A chain of transmission losses within the sourcepath-receiver system was applied to enable it to be applied to different situations. Similarly in [8] a semi-empirical method was presented based on a large number of measurements in Norway and Sweden. In another recent study, thousands of ground-borne vibration records across seven European countries were used in the analysis of the most influential 
parameters [9]. It was found in [9] that the soil properties are the most influential parameters, but these cannot normally be taken into account parametrically in empirical models.

The reliability of predictions based on these empirical approaches depends highly on the accuracy of measurements and the similarity to the locations covered by the database. Consequently, theoretical models offer a more efficient and flexible approach. These may be based on either analytical or numerical models.

Analytical models have the advantage of being rapid and computationally efficient. In [10] a three-dimensional analytical model was successfully developed based on a cylindrical tunnel embedded in an unbounded ground. Based on this model, Hussein et al. [11] created a user-friendly software called PiP (Pipe-in-pipe), which gives promising prediction results of the ground vibration induced by a train running in a tunnel. Nevertheless, this model is limited to simple circular tunnel structures and for simplicity neglects the motion of the train. Although the ground surface and ground layering can be introduced [11], there are some limitations to this approach, which is therefore mostly useful for scoping studies. Besides, the model cannot adequately take account of situations where the tunnel is too close to the ground surface or is directly beneath the building structure.

For more complex situations, and to allow increased flexibility, a numerical approach is preferred. With the development of high-performance computers, complex transmission problems can be solved more effectively and realistically. The finite element (FE) method is commonly used. When using FE models it is important that the boundaries of the finite computational domain do not introduce false reflections. To overcome this, if the FE approach is used to model the ground, Infinite Elements (IE) $[12,13,14]$ or Perfectly Matched Layers (PML) [15] can be adopted to terminate the FE mesh, allowing the reflections from the boundaries to be minimised.

Alternatively, the boundary element (BE) method can be used, as this includes the radiation condition implicitly. This can be used either on its own or coupled with the FE method. The coupled FE-BE method has the advantage that the structures near the track can be modelled in detail using FE, whereas homogeneous regions of soil can be represented more efficiently by BE. A two-dimensional (2D) model was developed using a combination of FE and BE methods by Jones et al. [16] and applied to study railway tunnels. The effects of design alterations of a bored tunnel and a cut-and-cover tunnel on the transmission properties of ground vibration were studied. Coupled FE-BE models for tunnels in two dimensions (2D) and three dimensions (3D) were proposed and compared in [17]. It was 
recommended that the $3 \mathrm{D}$ model should be used for the prediction of absolute vibration levels, although it involved a much higher computational cost.

As a compromise, the so-called two-and-a-half dimensional (2.5D) FE-BE method can provide results allowing for 3D effects but with significantly reduced model size. In this approach, a 2D FE-BE mesh is used but the problem is solved for a range of wavenumbers in the third dimension. The full 3D solution can be recovered from an inverse Fourier transform over wavenumber. Yang and Hung used such a 2.5D method with a finite/infinite element approach [18]. A 2.5D BE method for ground vibration was presented by Jean et al. [19] and later extended to include FE domains [20]. Coupled 2.5D FE-BE models have been applied to tunnels in [21] and surface trains in [22,23,24,25]. Similarly, a 2.5D FE-IE approach was proposed by Yang et al. and used to study ground vibration caused by a train in a tunnel [26,27]. Hung et al. introduced the roughness excitation using a $2.5 \mathrm{D}$ approach [28]. In these $2.5 \mathrm{D}$ approaches, it has to be assumed that the geometry and material properties are invariant in the length direction. More recently, other 2.5D coupled models based on the same concept have been introduced, such as the method of fundamental solutions - finite element method (MFS-FEM) [29] and the finite element method - scaled boundary finite element method (FEM-SBFEM) [30].

An alternative approach was adopted in [31] based on a periodic FE-BE model, in which the Floquet transform was employed to form the geometry of the tunnel. This approach allowed the periodicity of the tunnel segments of a cast-iron tunnel lining to be taken into account. The deformation modes of the tunnel structure were analysed and the effect of adding the soil on the dispersion results was investigated. However, this method involves more computational effort than the $2.5 \mathrm{D}$ approach.

In this paper, a numerical modelling approach for the prediction of train-induced vibration from trains in tunnels is described based on the 2.5D FE-BE method and comparisons are made with the results of field measurements. The 2.5D FE-BE approach is implemented in the software WANDS (Wave Number Domain Software) [32] which is introduced in Section 2. In contrast to the approach of $[22,23,24]$, in which the Green's functions of the ground include the effect the ground surface and layering, in the present case the boundary elements are for a homogeneous full-space. This allows greater flexibility in modelling ground geometry and allows non-parallel layering for example. The cross-section of the tunnel is modelled by using finite elements and the ground by boundary elements while the third direction is represented in the wavenumber domain. A particular issue for the tunnel studied here is that the tunnel structure is not homogeneous in the axial 
direction. An equivalent homogeneous structure is developed in Section 3 using finite element analysis of the free tunnel structure. Section 4 describes the coupling of this model with a train consisting of a series of vehicles, each represented by a 10 degree-of-freedom (DOF) multi-body model. The unevenness of the rail and wheel surface is used as the excitation to determine the dynamic contact forces between them. Then the train-induced vibration is determined by using these forces in combination with the transfer functions obtained from the tunnel-ground model. The quasi-static excitation due to the moving axle loads is also considered.

In Section 5 a validation measurement campaign is described and the results of the numerical model are compared with the measured results. These were obtained at a location in London where the tunnel depth from the ground surface to the tunnel crown is about 20 $\mathrm{m}$. Measurements were obtained of the vibration of the rail, the tunnel invert and tunnel wall. Additionally, simultaneous measurements were taken of the vibration on the ground surface above the tunnel during the passage of trains. These measurement results are compared with the predictions obtained using the coupled 2.5D FE-BE tunnel-ground model.

\section{Modelling method}

The prediction model is based on the waveguide finite element and boundary element method, known as the $2.5 \mathrm{D}$ approach. This is an efficient way of modelling the dynamic behaviour of structures with complex geometry but which can be assumed to be infinitely long and uniform in one direction. In the present application, the cross-section of the tunnel and track are modelled using finite elements and the soil is modelled using boundary elements. The responses within a two-dimensional cross-section are then determined for various wavenumbers in the longitudinal direction. The dependence of the responses on the third dimension can be recovered by an inverse Fourier transform over wavenumber. In this section, the theory of this method is introduced, based on $[21,32]$.

\subsection{General procedure}

In the 2.5D FE / BE method, the cross-section, which is assumed to be invariant in the longitudinal direction, is divided into several elements surrounded by nodes. The crosssection is defined by the coordinates $(y, z)$, while the longitudinal direction is the $x$ direction. Time-harmonic motion is assumed with circular frequency $\omega$ and time- 
dependence $e^{\mathrm{i} \omega t}$ while the dependence on $x$ is assumed to be $e^{-\mathrm{i} \beta x}$ where $\beta$ is the wavenumber.

The displacement amplitudes at each node are expressed as three translations $(u, v$ and $w$ ), corresponding to the displacement in $x, y$ and $z$ directions. For each element the displacements at all nodes are assembled into a vector $\mathbf{u}_{e}$. The displacements $\mathbf{q}_{e}$ at any position $(y, z)$ within the element are defined in terms of the displacements at the nodes of that element:

$$
\tilde{\mathbf{q}}_{e}(y, z)=\boldsymbol{\Phi}_{e} \tilde{\mathbf{u}}_{e}
$$

where the matrix $\boldsymbol{\Phi}_{e}$ contains the shape functions of the element and the tilde indicates the variables in the wavenumber domain. The size of the shape function matrix $\boldsymbol{\Phi}_{e}$ depends on the number of nodal degrees of freedom of the element.

Use is made of the following Fourier transform

$$
\mathbf{A}(k \beta, y, z)=\int_{-\infty}^{\infty} \mathbf{u}(x, y, z) e^{\mathrm{i} \beta x} \mathrm{~d} x
$$

where $\beta$ is the wavenumber in the $x$ direction. This allows the equations of motion to be written in terms of the wavenumber. Then the equations for all the elements are assembled into a global matrix equation. By inverting this matrix the vector of displacements on all the nodes of the model (u) can be determined for each wavenumber. The full 3D solution can be obtained by using the inverse Fourier transform:

$$
\mathbf{u}(x, y, z)=\frac{1}{2 \pi} \int_{-\infty}^{\infty} \mathbf{G}(k \beta, y, z) e^{-\mathrm{i} \beta x} \mathrm{~d} \beta
$$

For each element type, the equations of motion are obtained separately, and assembled to form a separate domain. Different domains are joined by the implementation of coupling conditions. The various types of element used in the current work and the method used to couple them are described briefly in the following.

\subsection{Solid finite elements}

Solid regions can be defined in the WANDS software by triangular or quadrilateral elements with linear, quadratic or cubic shape functions.

After assembling the matrices associated with different derivatives with respect to $x$, the equation of motion for the element can be expressed as [21]: 


$$
\left(\mathbf{K}_{s 2} \frac{\partial^{2}}{\partial x^{2}}+\mathbf{K}_{s 1} \frac{\partial}{\partial x}+\mathbf{K}_{s 0}-\omega^{2} \mathbf{M}_{s}\right) \mathbf{u}_{e}(x)=\mathbf{F}(x)
$$

where $\mathbf{K}_{s 2}, \mathbf{K}_{s 1}, \mathbf{K}_{s 0}$ and $\mathbf{M}_{s}$ are the stiffness and mass matrices for the solid element. $\mathbf{F}(x)$ is the vector of forces acting on the nodal degrees of freedom (DOF). Taking the Fourier transform of this equation gives

$$
\left(-\beta^{2} \mathbf{K}_{s 2}-\mathrm{i} \beta \mathbf{K}_{s 1}+\mathbf{K}_{s 0}-\omega^{2} \mathbf{M}_{s}\right) \mathbf{H}{ }_{e}(\beta)=\mathbf{F} \varphi(\beta)
$$

In the equation above, a dependence $e^{-\mathrm{i} \beta x}$ on both sides of the equation is implied. These mass and stiffness matrices are obtained for individual elements. The global matrices of the FE model are then assembled using conventional finite element techniques [33]. For each frequency $\omega$ and wavenumber $\beta$, Eq. (5) can then be written as:

$$
\left(\mathbf{K}_{\mathrm{fe}}(\beta)-\omega^{2} \mathbf{M}_{\mathrm{fe}}\right) \boldsymbol{A}_{\mathrm{qe}}=\mathbf{F}_{\mathrm{fe}}
$$

in which the subscript fe denotes the whole finite element domain.

\subsection{Beam elements}

Beams aligned with the $x$ axis can be represented by a single node. These are used in the present model for the rails. These elements include three types of motion: flexure, extension and torsion. The flexural motion corresponds to bending waves propagating along a Euler-Bernoulli beam, which has the following equation of motion for the displacement $w$ in the $z$ direction:

$$
D_{z} \frac{\partial^{4} w}{\partial x^{4}}-\omega^{2} m_{a} w=F_{z}
$$

where $m_{a}=\rho A$ is the mass per unit length in the longitudinal direction, $D_{z}$ is the flexural rigidity for bending about the $y$-axis, and $F_{z}$ is the external force in the $z$-direction. The equation of motion for flexure in the $y$ direction $(v)$ is similar. The coupling between the two motions can also be included but is not considered here.

The equations of motion for the longitudinal and torsional motion are

$$
\begin{aligned}
& D_{l} \frac{\partial^{2} u}{\partial x^{2}}-\omega^{2} m_{a} u=F_{x} \\
& D_{t} \frac{\partial^{2} \phi}{\partial x^{2}}-\omega^{2} J_{a} \phi=M_{x}
\end{aligned}
$$

where $D_{l}$ and $D_{t}$ are the axial rigidity and the torsional rigidity about the $x$-axis, $J_{a}$ is the polar second moment of area, and $F_{x}$ and $M_{x}$ are the corresponding force and moment. 
The four equations of motion above can be combined into a matrix equation

$$
\left(\mathbf{K}_{b 4} \frac{\partial^{4}}{\partial x^{4}}+\mathbf{K}_{b 2} \frac{\partial^{2}}{\partial x^{2}}-\omega^{2} \mathbf{M}_{b}\right) \mathbf{u}_{e}=\mathbf{F}_{b}
$$

where $\mathbf{u}_{e}$ contains the three displacements $u, v$ and $w$ and the rotation $\phi$ about the $x$ axis. $\mathbf{K}_{b 4}, \mathbf{K}_{b 2}$ and $\mathbf{M}_{b}$ denote the stiffness and mass matrices of the beam element, which can readily be assembled from Eq.(7) (9). $\mathbf{F}_{b}$ represents the force vector assembled from $F_{x}, F_{y}$, $F_{z}$ and $M_{x}$. Although not used here, a similar equation is implemented in the software for plate elements, which have two nodes, but in this case they also contain the stiffness matrices corresponding to the first and zero order derivatives $\mathbf{K}_{1}, \mathbf{K}_{0}$.

Taking the Fourier transform of Eq. (10), the equation of motion of the beam element can be written in terms of wavenumber as:

$$
\left(\beta^{4} \mathbf{K}_{b 4}-\beta^{2} \mathbf{K}_{b 2}-\omega^{2} \mathbf{M}_{b}\right)\{\boldsymbol{\mathbf { U }} b\}=\mathbf{F}_{b}^{o}
$$

The coupling of the beam element to the solid finite element is achieved by adding the stiffness and mass matrices of the coupled beam element into Eq.(5), making use of the transformation matrix $\mathbf{T}_{s b}$ :

$$
\left(\beta^{4} \mathbf{K}_{s b 4}-\beta^{2}\left(\mathbf{K}_{s 2}+\mathbf{K}_{s b 2}\right)-\mathrm{i} \beta \mathbf{K}_{s 1}+\mathbf{K}_{s 0}-\omega^{2}\left(\mathbf{M}_{s}+\mathbf{M}_{s b}\right)\right)\left\{\mathbf{w} o_{e}\right\}=\mathbf{F}_{s}^{\circ}
$$

where $\mathbf{K}_{s b 4}=\mathbf{T}_{s b}{ }^{T} \mathbf{K}_{b 4} \mathbf{T}_{s b}$ and similarly for $\mathbf{K}_{s b 2}$ and $\mathbf{M}_{s b} . \mathbf{T}_{s b}$ transforms the beam degrees of freedom onto those of the solid element mesh, in terms of the location of the beam element between the two adjacent nodes of the solid element.

\subsection{Boundary elements}

A homogeneous elastic body can be defined by the boundary $\Gamma$ around it, which can be discretised using boundary elements. In the current implementation [32], which uses fullspace Green's functions, the ground surface is represented by a BE mesh extending out to a certain distance. As this is, of necessity, truncated to a finite width, the edges of the boundary element mesh are specially treated to minimise the reflection of waves [21].

For the boundary $\Gamma$, discretized into 3-noded boundary elements, the equation of motion can be assembled as [21]:

$$
\mathbf{H}_{\mathrm{be}} \mathbf{A}_{\mathrm{be}}+\mathbf{G}_{\mathrm{be}} \mathbf{p}_{\mathrm{be}}=0
$$

where $\boldsymbol{f}_{\mathrm{be}}$ and $\boldsymbol{p}_{\mathrm{be}}$ are the displacement and traction vectors of all the nodal DOFs of the BE domain. The matrices $\mathbf{H}_{\mathrm{be}}$ and $\mathbf{G}_{\mathrm{be}}$ are square and fully populated and depend on both the 
frequency $\omega$ and the wavenumber $\beta$. Further details are given in [34]. In the BE formulation, body forces can also be applied on each node, but these are not considered here.

Boundary conditions are also required, which can be expressed as:

$$
\mathbf{C}_{A} \mathbf{t}_{b \mathrm{e}}+\mathbf{C}_{B} \boldsymbol{p}_{\mathrm{be}}=\mathbf{C}_{C}
$$

The coefficients $\mathbf{C}_{A}, \mathbf{C}_{B}$ and $\mathbf{C}_{c}$ depend on the local boundary conditions, which may be external constraints or coupling to the other domains. For example, for a free surface, the traction is set to zero.

\subsection{Coupling between domains}

The coupling between different domains ( $\mathrm{FE}$ to $\mathrm{BE}$ or $\mathrm{BE}$ to $\mathrm{BE}$ ) is similar to the assembly of the global equations. However, when coupling the domains with different element types, as they are established in different local coordinate systems, a transformation matrix is required to convert the displacement and force vectors into a common global coordinate system. Moreover, to achieve a flexible approach, the coupling is implemented in WANDS by using separate coupling equations rather than by eliminating common DOFs [32].

The coupling of a BE domain (subscript 1) to another (subscript 2) is treated in terms of boundary conditions. The terms $\mathbf{C}_{A}, \mathbf{C}_{B}$ in Eq. (14) are replaced by $\mathbf{A}_{1}, \mathbf{B}_{1}$ and $\mathbf{A}_{2}, \mathbf{B}_{2}$ and used to represent compatibility of displacements and equilibrium of tractions on the coupled boundary. Then, the coupled equation can be expressed as:

$$
\left[\begin{array}{cccc}
\mathbf{G}_{1} & \mathbf{H}_{1} & \mathbf{0} & \mathbf{0} \\
\mathbf{0} & \mathbf{0} & \mathbf{G}_{2} & \mathbf{H}_{2} \\
\mathbf{A}_{1} & \mathbf{B}_{1} & \mathbf{A}_{2} & \mathbf{B}_{2}
\end{array}\right]\left\{\begin{array}{l}
\boldsymbol{p}_{\varphi} \\
\mathbf{A}_{\varphi} \\
\boldsymbol{p}_{2} \\
\mathbf{U}_{2}
\end{array}\right\}=\left\{\begin{array}{l}
\mathbf{0} \\
0 \\
0
\end{array}\right\}
$$

To couple an FE domain to a BE domain, the force on the boundary shared with the $\mathrm{BE}$ domain is added to the equation of the FE domain:

$$
\left(\mathbf{K}_{\mathrm{fe}}(\beta)-\omega^{2} \mathbf{M}_{\mathrm{fe}}\right) \mathbf{d}_{\mathrm{fe}}-\mathbf{T}_{1} \boldsymbol{\beta}_{\mathrm{be}}^{\prime}=\mathbf{F}_{\mathrm{fe}}^{O}
$$

where $\mathbf{T}_{1}$ transfers the tractions from local nodes of the boundary elements to the finite element DOFs. An additional constraint equation is required to ensure compatibility of displacements of the two domains:

$$
\mathbf{I}_{\mathrm{be}}-\mathbf{T}_{2} \mathbf{A}_{\mathbf{P}_{\mathrm{e}}}=\{\mathbf{0}\}
$$

where $\mathbf{I}$ is a unit matrix and $\mathbf{T}_{2}$ is used to map the displacements from local nodes of the finite elements to those of the boundary elements. Hence, the coupled equation can be assembled as: 


$$
\left[\begin{array}{ccc}
\left(\mathbf{K}_{\mathrm{fe}}(\beta)-\omega^{2} \mathbf{M}_{\mathrm{fe}}\right) & -\mathbf{T}_{1} & \mathbf{0} \\
-\mathbf{T}_{2} & \mathbf{0} & \mathbf{I} \\
\mathbf{0} & \mathbf{G} & \mathbf{H}
\end{array}\right]\left\{\begin{array}{l}
\boldsymbol{U}_{\mathrm{fe}} \\
\boldsymbol{P}_{\mathrm{be}}^{b} \\
\mathbf{U}_{\mathrm{be}}
\end{array}\right\}=\left\{\begin{array}{c}
\boldsymbol{F}_{\mathrm{fe}}^{o} \\
\mathbf{0} \\
\mathbf{0}
\end{array}\right\}
$$

\subsection{Solution}

Once the complete system matrices have been assembled, the vector of nodal displacements and tractions can be readily solved for each frequency $\omega$ and wavenumber $\beta$ by inverting the system matrix. To recover the solution in the longitudinal direction it is then necessary to perform a numerical integration over discrete wavenumbers to approximate Eq. (3). These are defined within a certain wavenumber range $\left[-\beta_{\max } \beta_{\max }\right]$ with an equal spacing of $\Delta \beta$ :

$$
\hat{\mathbf{u}}(x, y, z, \omega)=\frac{1}{2 \pi} \sum_{-\beta_{\max }}^{\beta_{\max }} \mathbf{A}(k \beta, y, z, \omega) e^{-\mathrm{i} \beta x} \Delta \beta
$$

The choice of the maximum wavenumber $\left(\beta_{\max }\right)$ and the wavenumber resolution $(\Delta \beta)$ used in the calculation will affect the accuracy of the results. Therefore, the calculation should be carried out over a reasonable wavenumber range so that the numerical integration can best approach the actual results.

The relationships between the variables $\beta_{\max }$ and $\Delta \beta$ in the wavenumber domain and the corresponding ones in the spatial domain are:

$$
\begin{gathered}
\Delta x=\frac{\pi}{\beta_{\text {max }}} \\
x_{\text {min }}=-\frac{\beta_{\text {max }}}{\Delta \beta} \Delta x \quad x_{\text {max }}=\left(\frac{\beta_{\text {max }}}{\Delta \beta}+1\right) \Delta x
\end{gathered}
$$

where $x_{\min }$ and $x_{\max }$ are the start and end values of the range of $x$ considered.

After applying the inverse Fourier transform, Eq. (19), the results from the $2.5 \mathrm{D}$ $\mathrm{FE} / \mathrm{BE}$ model take the form of complex displacement amplitudes at a set of equally spaced positions along the $x$ axis on the nodes of the cross-section. A unit point force acting at $x=0$ can be simulated by applying a unit force at every wavenumber.

An alternative solution method for 2.5D FE analysis, described in [35], is to use residue calculus to determine the Fourier transform integral. This relies on first obtaining the eigenvalues of the dispersion relation, Eq. (6). However, although this is effective for a finite element calculation, it cannot be used when boundary element domains are included in the 
model as the boundary element equation, Eq. (13), involves element integrals that must be determined for each wavenumber. It cannot therefore be solved as an eigenvalue problem.

In this paper, the 2.5D FE / BE method described in this section is adopted to model the ground using BE and the structures (track and tunnel) using FE, which, to differing extent, can be regarded as having uniform properties along the track direction.

\section{Equivalent tunnel model}

The tunnel, for which measurements are presented in Section 5, is shown in Figure 1. This is a cast-iron structure made up of seven segments around the circumference, which are bolted together at circular end flanges. The segments are $0.5 \mathrm{~m}$ long in the axial direction and again are bolted together at their flanges. These flanges provide additional stiffeners in both the circumferential and the longitudinal directions. These discrete stiffeners cannot be modelled using the 2.5D approach. In [31] a periodic structure approach was adopted to represent this structure, but here an alternative approach is investigated in which an equivalent isotropic cylinder is used.

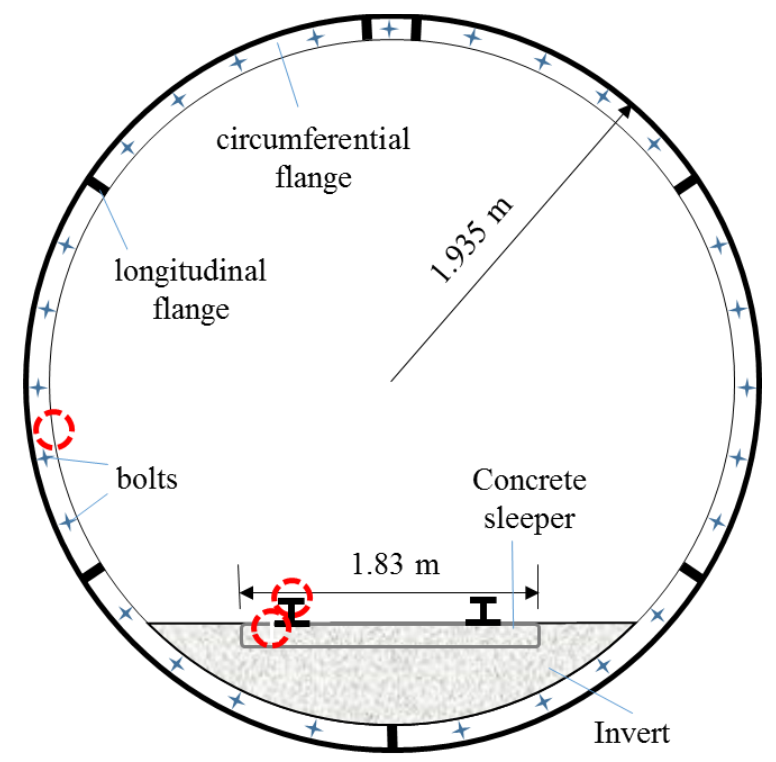

Figure 1 Cross-section of the metro tunnel. Circles indicate measurement locations.

To obtain the equivalent isotropic cylinder, the dynamic behaviour of the cast-iron tunnel, without the ground, is studied using a 3D finite element model in ANSYS including both longitudinal and circumferential stiffeners. The length of the model is $15 \mathrm{~m}$ and at the two ends symmetric and/or anti-symmetric displacement constraints are applied. The use of these boundary conditions ensures that the mode shapes are approximately sinusoidal along the axial direction. By identifying the number of wavelengths in the length of the model in 
each mode shape, the axial wavenumber corresponding to each wave mode can be determined. These are plotted against the natural frequencies in Figure 2, shown as symbols. The dispersion curves plotted as lines, are obtained from the equivalent isotropic tunnel model calculated using the 2.5D FE model in WANDS. The method of obtaining the equivalent isotropic tunnel models is described as below.

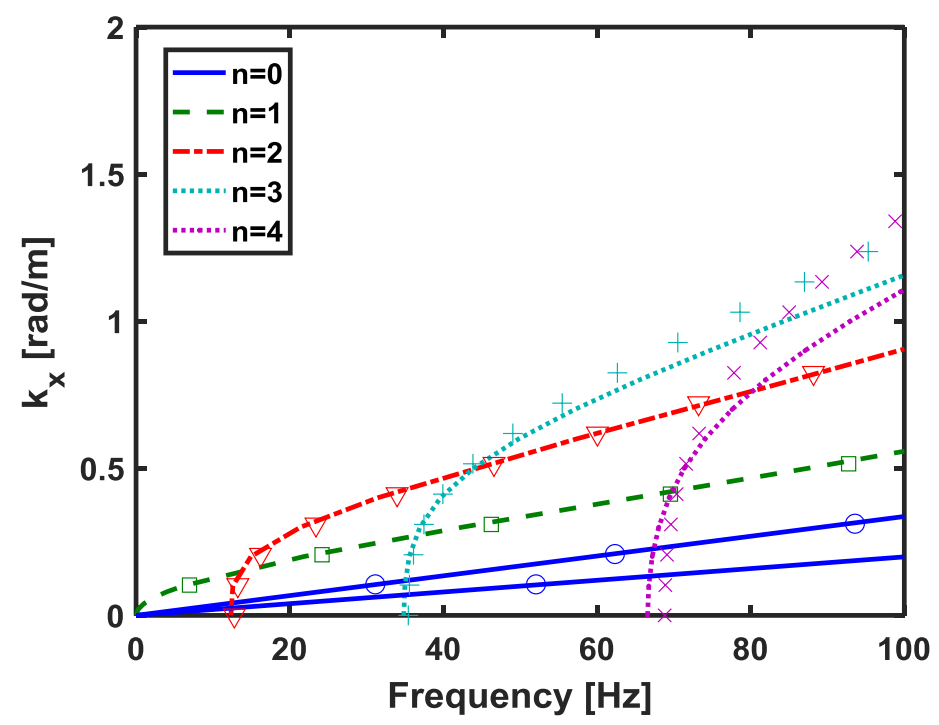

Figure 2 Dispersion diagram (symbols: 3D FE model; lines: 2.5D equivalent model)
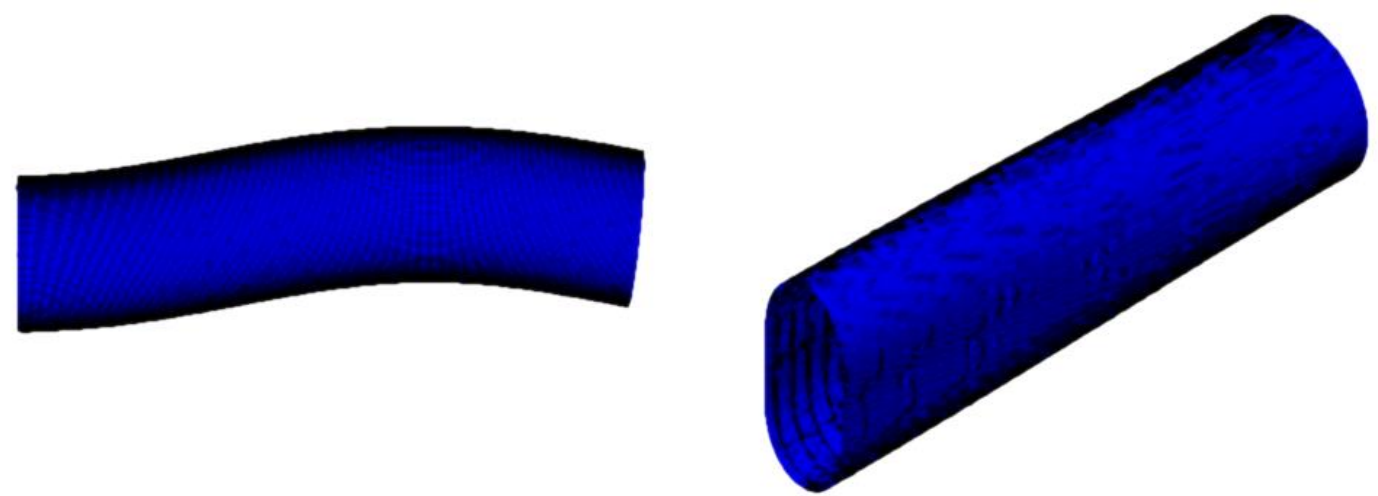

(a)

(b)

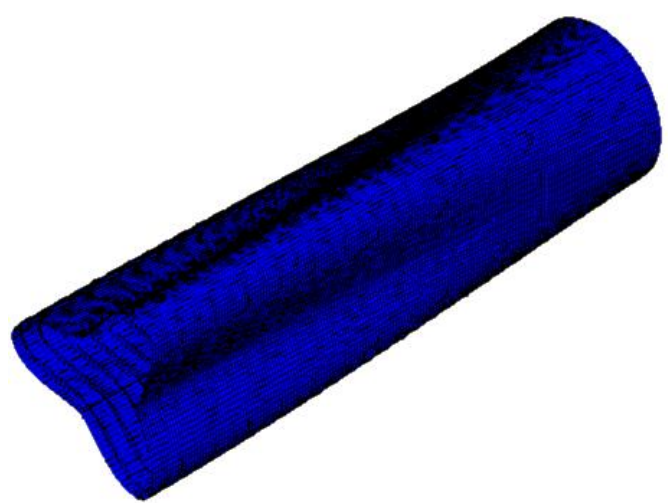

(c)

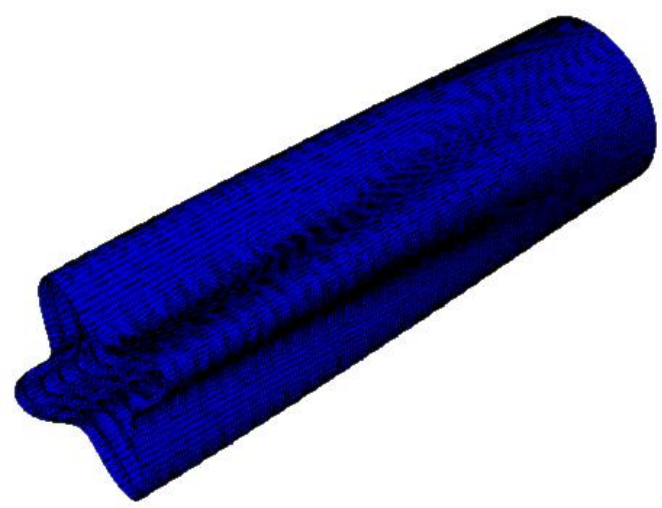

(d)

Figure 3 Examples of out-of-plane deformation modes from 3D ANSYS model. (a) $n=1, f=46 \mathrm{~Hz}$; (b) $n=2, f=13 \mathrm{~Hz}$; (c) $n=3, f=36 \mathrm{~Hz}$; (d) $n=4, f=69 \mathrm{~Hz}$. 
Examples of the mode shapes for circumferential orders $n=1 \sim 4$ are shown in Figure 3. The $n=0$ modes, not shown in Figure 3, correspond to the axial and torsional modes. The $n=1$ modes represent bending waves in which the whole tunnel behaves like a Timoshenko beam. This is the most influential mode in the radiation of vibration into the ground.

In determining the parameters of the equivalent isotropic cylinder, it is recognised that it is not possible to find a set of parameters for which all the modes match the FE results completely, especially the higher order modes at high frequencies. The strategy adopted is to fit the most influential modes $(n=0,1)$ as closely as possible while also matching the cut-on frequencies of the higher order modes. The parameters which are adjusted are the Young's modulus $E$, density $\rho$ and thickness $h$ of the isotropic cylinder.

The wavenumbers of the longitudinal $\left(k_{L}\right)$ and torsional $\left(k_{T}\right)$ waves in a circular elastic beam can be obtained from

$$
k_{L}=\omega \sqrt{\frac{\rho}{E}} ; \quad k_{T}=\omega \sqrt{2(1+v) \frac{\rho}{E}}
$$

where $\omega=2 \pi f$ is the angular frequency. $v$ is Poisson's ratio which is assumed to be 0.3 . The ratio between the equivalent Young's modulus $(E)$ and density $(\rho)$ can then be estimated from the dispersion curves for $n=0$ shown in Figure 2, giving a value of $E / \rho=9 \times 10^{6} \mathrm{~m}^{2} / \mathrm{s}^{2}$. This is independent of the wall thickness.

Based on Donnell's thin shell theory [36], for modes $n>1$, the cut-on frequency can be approximated by

$$
f_{n} \approx n^{2} \alpha f_{0}
$$

where $\alpha^{2}=h^{2} / 12 a^{2}, h$ is the wall thickness and $a=r-h / 2$ is the radius of the middle line. $r$ is the outer radius of the isotropic cylinder, which is kept the same as the original tunnel, i.e. $r=1.953 \mathrm{~m} . f_{0}$ is the 'ring frequency', defined as

$$
f_{0}=\frac{1}{2 \pi a} \sqrt{\frac{E}{\rho\left(1-v^{2}\right)}}
$$

According to the cut-on frequencies of modes $n=2,3$ and 4 shown in Figure 2, the average value of $\alpha f_{0}$ can be estimated from Eq. (23) as 3.84 .

Combining this with the ratio $E / \rho$ obtained from Eq. (22), the equivalent wall thickness can be found $(h=0.114 \mathrm{~m})$. Keeping the total tunnel mass constant, the equivalent density can then be obtained and subsequently the equivalent Young's modulus. The equivalent parameters obtained are shown in Table 1. 
Table 1 Material properties of tunnel structure

\begin{tabular}{|c|c|c|c|}
\hline & Young's Modulus & Poisson's ratio & Density \\
\hline Cast Iron (Grade 20) & $100 \mathrm{GPa}$ & 0.3 & $7150 \mathrm{~kg} / \mathrm{m}^{3}$ \\
\hline Equivalent Isotropic Cylinder & $19.1 \mathrm{GPa}$ & 0.3 & $2104 \mathrm{~kg} / \mathrm{m}^{3}$ \\
\hline
\end{tabular}

Although these parameters are obtained using thin shell theory, they are subsequently used in the 2.5D FE model, which does not have the same limitations as the thin shell theory. In the comparison of the resulting dispersion relations in Figure 2 between the 3D FE model including the stiffeners and the 2.5D FE model of the equivalent cylinder, it can be seen that good agreement is obtained for the axial, torsional and bending modes. Moreover, for the higher order modes, the dynamic behaviour shows approximately the same cut-on frequencies and dispersion at low wavenumbers. The divergence at higher wavenumbers and frequencies is expected to be less significant for the case of the tunnel embedded in the ground.

\section{Assembly of the prediction model}

The track, tunnel and ground are modelled with the $2.5 \mathrm{D}$ method described in Section 2 using the WANDS software [32], whereas the train is represented by multi-body vehicle models. The two parts are coupled together, using the unevenness of the rail as the excitation, to calculate the dynamic component of vibration induced by the train. The quasistatic component is obtained separately based on the response to a unit excitation on the rail which also obtained from the WANDS model. The details of the vehicle model, the coupling method and the estimate of the quasi-static component are explained in the following sub-sections.

\subsection{Train model}

The train is treated as multiple independent vehicles, ignoring the connections between them. Each vehicle is represented as a 10-DOF multi-body model, as shown in Figure 4(a). This is a conventional two-dimensional vehicle model that includes four wheelsets and two bogies. Only vertical motion is included, as well as the pitching motion of the car body and bogies. A sketch of a single vehicle is also shown in Figure 4(b) to indicate the main dimensions. $l_{w}, l_{b}$ and $l_{c}$ indicate half the distances between the wheelsets, bogies and vehicles, respectively. 


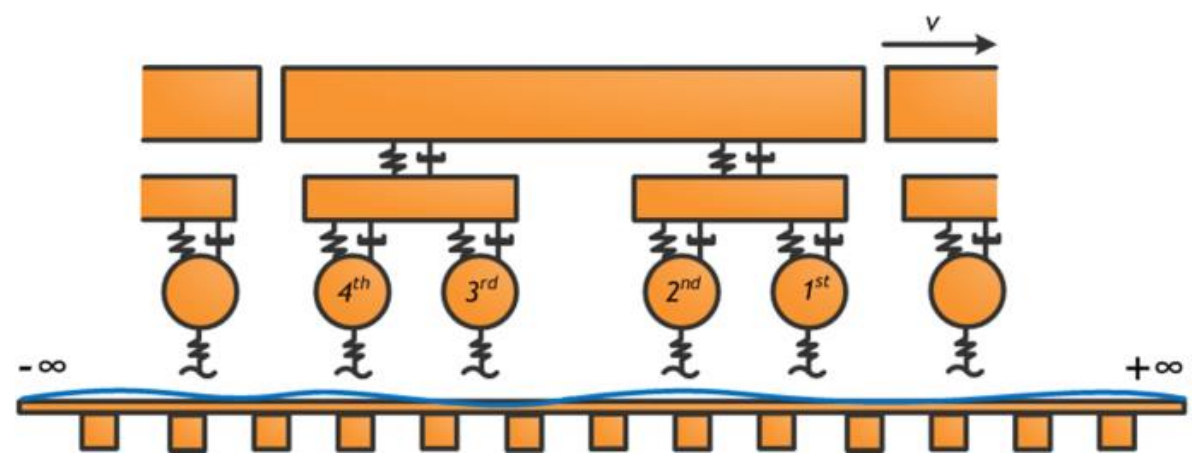

(a)

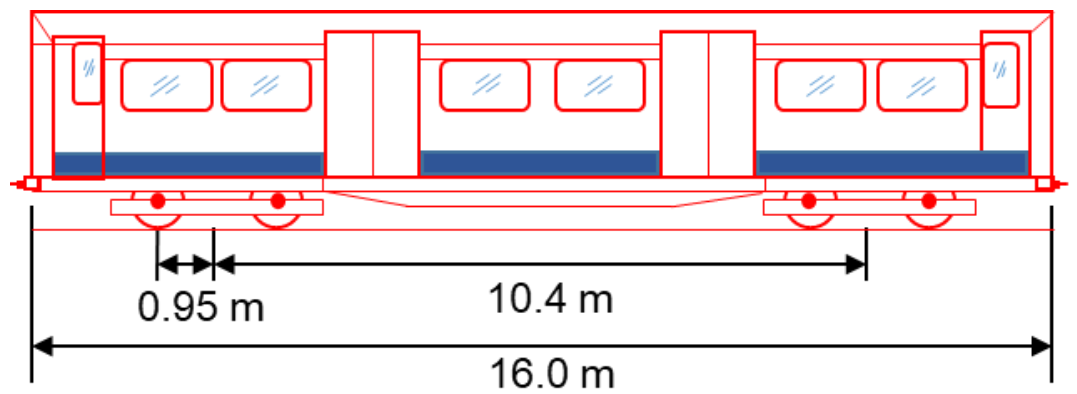

(b)

Figure 4 (a) the coupled track-vehicle model and (b) the dimensions of a vehicle

For each vehicle, the degrees of freedom are

$$
\mathbf{u}=\left[\begin{array}{llllllllll}
w_{1} & w_{2} & w_{3} & w_{4} & w_{b 1} & \theta_{b 1} & w_{b 2} & \theta_{b 2} & w_{c} & \theta_{c}
\end{array}\right]^{T}
$$

where $w_{1}, \ldots w_{4}$ are the vertical displacements of the wheelsets, $w_{b 1}$ and $w_{b 2}$ are the vertical displacements of the bogies, $\theta_{b 1}$ and $\theta_{b 2}$ are the bogie pitch rotations, and $w_{c}$ and $\theta_{c}$ are the displacement and pitch rotation of the car body. The mass matrix of each vehicle can be formed as:

$$
\mathbf{M}=\operatorname{diag}\left[\begin{array}{llllllllll}
m_{w} & m_{w} & m_{w} & m_{w} & m_{b} & J_{b} & m_{b} & J_{b} & m_{c} & J_{c}
\end{array}\right]
$$

The symbols and corresponding values are defined in Table 2 . The stiffness matrix is

$$
\mathbf{K}=\left[\begin{array}{cccccccccc}
k_{s 1} & 0 & 0 & 0 & -k_{s 1} & k_{s 1} l_{w} & 0 & 0 & 0 & 0 \\
0 & k_{s 1} & 0 & 0 & -k_{s 1} & -k_{s 1} l_{w} & 0 & 0 & 0 & 0 \\
0 & 0 & k_{s 1} & 0 & 0 & 0 & -k_{s 1} & k_{s 1} l_{w} & 0 & 0 \\
0 & 0 & 0 & k_{s 1} & 0 & 0 & -k_{s 1} & -k_{s 1} l_{w} & 0 & 0 \\
-k_{s 1} & -k_{s 1} & 0 & 0 & 2 k_{s 1}+k_{s 2} & 0 & 0 & 0 & -k_{s 2} & k_{s 2} l_{b} \\
k_{s 1} l_{w} & -k_{s 1} l_{w} & 0 & 0 & 0 & 2 k_{s 1} l_{w}^{2} & 0 & 0 & 0 & 0 \\
0 & 0 & -k_{s 1} & -k_{s 1} & 0 & 0 & 2 k_{s 1}+k_{s 2} & 0 & -k_{s 2} & -k_{s 2} l_{b} \\
0 & 0 & k_{s 1} l_{w} & -k_{s 1} l_{w} & 0 & 0 & 0 & 2 k_{s 1} l_{w}^{2} & 0 & 0 \\
0 & 0 & 0 & 0 & -k_{s 2} & 0 & -k_{s 2} & 0 & 2 k_{s 2} & 0 \\
0 & 0 & 0 & 0 & k_{s 2} l_{b} & 0 & -k_{s 2} l_{b} & 0 & 0 & 2 k_{s 2} l_{b}^{2}
\end{array}\right]
$$


where $l_{b}$ is half the distance between bogie centres, and $l_{w}$ is half the distance between wheelsets in a bogie (see Figure 4(b)). The formulation of the damping matrix $\mathbf{C}$ is similar to K.

The displacements and rotations due to a unit force $\mathbf{F}_{j}$ at the $j$ th wheelset $(j=1,2,3,4)$ can be obtained from:

$$
\mathbf{u}=\left(-\omega^{2} \mathbf{M}+i \omega \mathbf{C}+\mathbf{K}\right)^{-1} \mathbf{F}_{j}
$$

where $\mathbf{F}_{j}$ is a force vector with the $j^{\text {th }}$ term set to unity. The displacements of the four wheelsets can be extracted from u. Multiplying by $\mathrm{i} \omega$, the wheel mobilities can be obtained. The $4 \times 4$ mobility matrix of the vehicle $\left(\mathbf{Y}_{w}^{\mathrm{veh}}\right)$ is assembled from the mobility of the four wheels due to a unit force acting at each wheel in turn. From this, the mobility matrix for the whole train is assembled as a block diagonal matrix containing $\mathbf{Y}_{w}^{\mathrm{veh}}$ :

$$
\mathbf{Y}_{w}=\left[\begin{array}{ccc}
\mathbf{Y}_{w}^{\mathrm{veh}} & & 0 \\
& \mathrm{O} & \\
0 & & \mathbf{Y}_{w}^{\mathrm{veh}}
\end{array}\right]_{4 n \times 4 n}
$$

where $n$ is the number of vehicles in the train.

\begin{tabular}{|c|c|c|}
\hline \multirow{2}{*}{ Car body } & $\operatorname{mass}\left(m_{w}\right)$ & $18200 \mathrm{~kg}$ \\
\hline & pitch moment of inertia $\left(J_{c}\right)$ & $0.4 \times 10^{6} \mathrm{~kg} \cdot \mathrm{m}^{2}$ \\
\hline \multirow{2}{*}{ Bogie } & $\operatorname{mass}\left(m_{b}\right)$ & $2096 \mathrm{~kg}$ \\
\hline & pitch moment of inertia $\left(J_{b}\right)$ & $630 \mathrm{~kg} \cdot \mathrm{m}^{2}$ \\
\hline \multirow{2}{*}{$\begin{array}{l}\text { Wheelset unsprung mass } \\
\qquad\left(m_{w}\right)\end{array}$} & Trailer car & $1220 \mathrm{~kg}$ \\
\hline & Motor car & $2308 \mathrm{~kg}$ \\
\hline \multirow{2}{*}{$\begin{array}{l}\text { Primary suspension per } \\
\text { wheelset }\end{array}$} & stiffness $\left(k_{s 1}\right)$ & $1.346 \mathrm{MN} / \mathrm{m}$ \\
\hline & viscous damping $\left(c_{s 1}\right)$ & $21.4 \mathrm{kNs} / \mathrm{m}$ \\
\hline \multirow{2}{*}{$\begin{array}{l}\text { Secondary suspension per } \\
\text { bogie }\end{array}$} & stiffness $\left(k_{s 2}\right)$ & $11.31 \mathrm{MN} / \mathrm{m}$ \\
\hline & viscous damping $\left(c_{s 2}\right)$ & $62.06 \mathrm{kNs} / \mathrm{m}$ \\
\hline \multicolumn{2}{|c|}{ Contact stiffness per wheelset $\left(k_{H}\right)$} & $2.926 \mathrm{GN} / \mathrm{m}$ \\
\hline \multicolumn{2}{|c|}{ Vehicle length } & $16 \mathrm{~m}$ \\
\hline \multicolumn{2}{|c|}{ Distance between bogie centres $\left(2 l_{b}\right)$} & $10.4 \mathrm{~m}$ \\
\hline \multicolumn{2}{|c|}{ Bogie wheelbase $\left(2 l_{w}\right)$} & $1.9 \mathrm{~m}$ \\
\hline
\end{tabular}

Table 2 Parameters of vehicle model

The assumed parameters of a single vehicle are listed in Table 2. The car body mass corresponds to the loading when the seats are fully occupied. Figure 5 shows the mobility of the 10-DOF vehicle (at the leading wheelset). As the trailer and motor cars have different 
unsprung masses (as shown in Table 2), the corresponding wheel mobilities are given separately.

\subsection{Track/tunnel/ground model}

The 2.5D track-tunnel-ground model is used to determine the mobility matrix $\left(\mathbf{Y}_{r}\right)$ of the track for forces applied at the position of each wheelset in turn. Vertical forces of magnitude 0.5 are applied to each of the two rails in phase, as the train is modelled as a series of $2 \mathrm{D}$ multi-body vehicles in the length direction and only symmetric motion is included. The point mobility of the track is obtained from the $2.5 \mathrm{D}$ tunnel/ground model and is shown in Figure 5. In this frequency range the track mobility is stiffness-controlled whereas the wheel mobility (above $10 \mathrm{~Hz}$ ) is mass-controlled. The resonance frequency of the coupled vehicle/track system can therefore be identified as the intersection of the wheel and rail mobilities, which occurs around $80-100 \mathrm{~Hz}$ as seen in Figure 5.

The track mobility matrix $\mathbf{Y}_{r}$ also contains transfer mobilities between each pair of wheelset positions. As the spacing between the wheelsets on the rail may not correspond exactly to the spatial resolution output from the $2.5 \mathrm{D}$ model (which is determined by the chosen wavenumber range), linear interpolation is employed to obtain the displacements at the exact locations required.

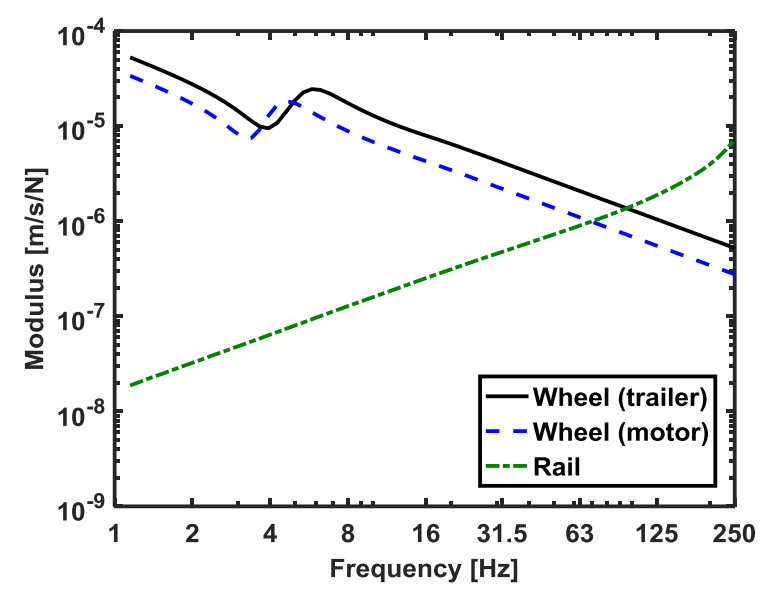

Figure 5 Point mobility of wheel and rail

The transfer mobilities from the track to the ground surface $\left(\mathbf{Y}_{g}\right)$ corresponding to excitation at the position of each wheelset in the train are also extracted from the 2.5D model. These are then used in the prediction of train-induced vibration at different monitor points. More details are given in the next section. 


\subsection{Coupling method for excitation due to roughness}

The dynamic component of vibration is caused by the train-track interaction, due to wheel/rail unevenness. To determine the dynamic component of vibration, the train and tunnel-ground models are coupled at the contact points between the wheel and rail, as shown in Figure 4(a). Considering excitation due only to the rail unevenness, the excitation of all the wheelsets can be assumed to be identical apart from a time lag related to the speed of train and the distances between them [37]. As a 2D vehicle model is used, it is assumed that the unevenness of the two rails is identical. In [38] it is shown that the roughness on the two rails should be treated as correlated at long wavelengths (greater than about $5 \mathrm{~m}$ ) but as only partially correlated at shorter wavelengths. However, the error introduced by assuming fully correlated inputs was found to be mostly less than $2 \mathrm{~dB}$. If the roughness amplitude at frequency $\omega$ at one wheel/rail position is $r$, the roughness amplitudes for all $n_{\mathrm{w}}$ wheel/rail positions can be written as $\mathbf{R}=\mathbf{T}_{r} \times r$, where $\mathbf{T}_{r}$ is the time delay vector [37]:

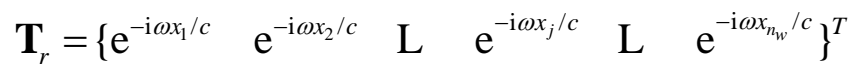

where $c$ is the speed of train and $x_{j}$ are the wheelset positions.

The vector of contact forces $\mathbf{F}_{c}$ between the rail and the wheelsets can be obtained by solving

$$
\left[\mathbf{Y}_{r}+\mathbf{Y}_{w}+\mathbf{Y}_{c}\right]_{4 n \times 4 n} \mathbf{F}_{c}=\mathrm{i} \omega \mathbf{R}
$$

where $\mathbf{Y}_{r}, \mathbf{Y}_{w}$ and $\mathbf{Y}_{c}$ are matrices of the mobilities of the rail, wheel and contact spring respectively. $\mathbf{Y}_{w}$ and $\mathbf{Y}_{r}$ are obtained from the train model and the tunnel-ground model as described in Section 4.1 and Section 4.2 respectively. $\mathbf{Y}_{c}=\left(\mathrm{i} \omega / k_{\mathrm{H}}\right) \mathbf{I}_{4 n \times 4 n}$ in which $k_{\mathrm{H}}$ is the stiffness of a linearised Hertzian spring, given in Table 2 which represents the sum of the stiffness applying to both wheels on a wheelset.

Using the contact force vector obtained from Eq. (31), the response $v_{g}$ at a receiver point due to the train can be obtained by

$$
v_{g}=\mathbf{Y}_{g} \mathbf{F}_{c}
$$

where $\mathbf{Y}_{g}$ is a vector of transfer mobilities from forces at different wheel positions on top of the rails to the receiver point, which is obtained from the WANDS model. To determine an average level over the train length, the response $v_{g}$ at different values of $x$ within the range of the train length is obtained. 


\subsection{Obtaining the quasi-static component}

The quasi-static component of vibration refers to the deflections under the constant axle loads moving with the passage of the train. This is distinct from the dynamic component of vibration described above and must be calculated separately.

As the 2.5D model is based on moving Green's functions [21,32], the response to a constant moving load (speed $c \neq 0$, frequency $f=0 \mathrm{~Hz}$ ) on the railheads can be calculated. This represents the quasi-static displacements caused by a non-fluctuating moving load. For example, the displacement on the rail due to a unit vertical force ( 0.5 on each rail) is shown in Figure 6(a) for a nominal load speed of $48 \mathrm{~km} / \mathrm{h}$. Similar to the dynamic output, linear interpolation is adopted to extract the displacement at each axle location, if required. For clarity, the figure only shows the response within $50 \mathrm{~m}$ of the load, although the calculation range is $\pm 200 \mathrm{~m}$.

The quasi-static displacement under each axle load is then obtained by multiplying the corresponding displacement due to the unit load with the total load on each wheelset, which is $140 \mathrm{kN}$ for the case investigated. After shifting the displacements under each axle in the $x$ direction according to the axle spacing, the displacements under all the axle loads are combined to form the quasi-static displacement caused by a moving train, as shown in Figure 6(b). Some uplift can be seen before and after the train which is caused by the discretisation of the wavenumber Fourier integral. However, this has negligible effect on the resulting frequency spectrum.
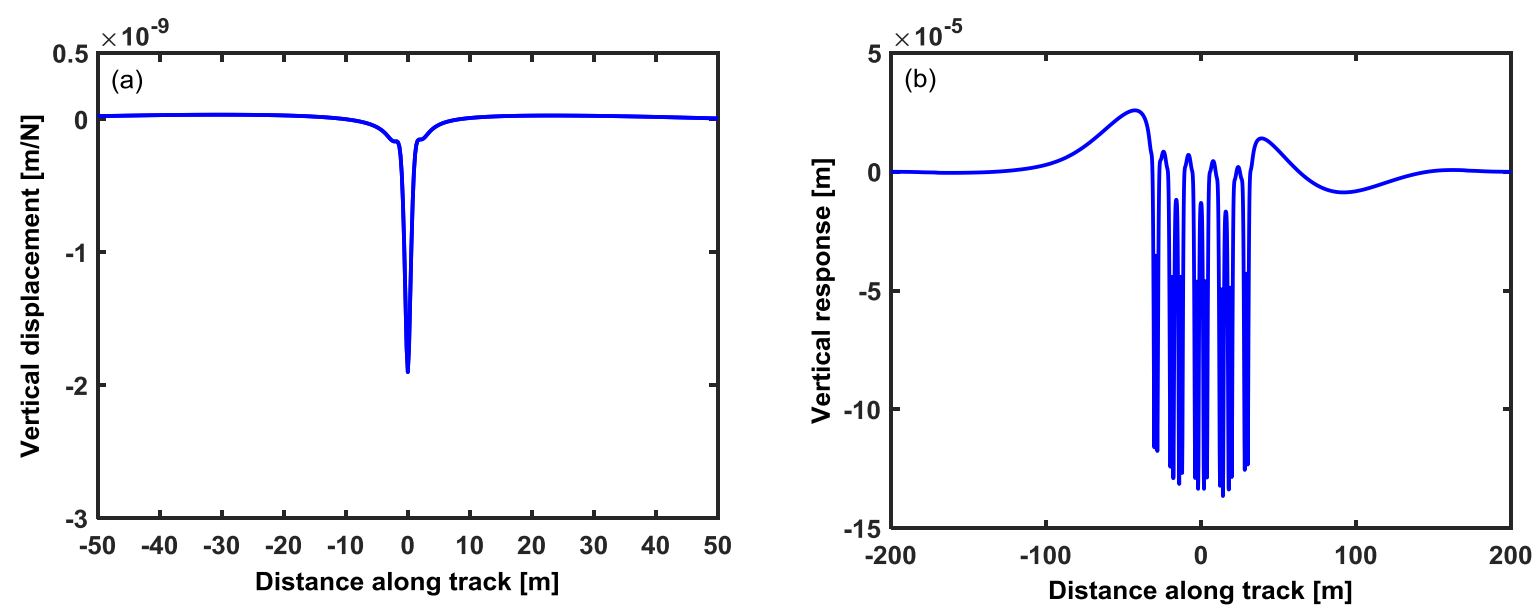

Figure 6 Rail response (a) due to a unit vertical load; (b) due to a four-vehicle train, in each case moving at $48 \mathrm{~km} / \mathrm{h}$.

In order to determine the spectrum of vibration at a fixed point as the train passes, as the results obtained from WANDS are given in the moving frame, the displacement as a function of time is obtained from the solution in the spatial domain by dividing the distance $x$ 
by the train speed $c$. After applying a Fourier transform, the spectrum of the quasi-static component of vibration in the frequency domain is obtained. The quasi-static vibration on the track and those in the far field are obtained in the same way.

\section{Results, analysis and comparison}

In this section, a series of measurements is presented and the results predicted using the coupled 2.5D model are compared with the measurement results.

\subsection{Measurements}

A series of measurements was carried out in June 2014 at a location in London on a conventional tube line. At the site, the tunnel crown is approximately $20 \mathrm{~m}$ below the street. The soil is London clay, the parameters of which were given in [31] for a site in Regents Park which is close to the current test site. The tunnel has a cast-iron structure as described in Section 3. The rails are laid on rail pads on concrete sleepers that are cast into the tunnel invert. A second tunnel runs parallel to the one under investigation but this has been neglected in the modelling. The effect of twin-tunnels has been addressed in [39], according to which it may be expected that the vibration level above the tunnel might be higher due to the existence of the second tunnel, especially at higher frequencies. However, no allowance is made for that in the current work.

Coordinated measurements took place in the tunnel and on the ground surface. The in-tunnel measurements included the train-induced vibration measured on the rail, the tunnel invert and the tunnel wall (see Figure 1). In each case accelerometers with a mass of $8.6 \mathrm{~g}$ were attached via a small mounting block (mass less than $50 \mathrm{~g}$ ) using a stiff acrylic adhesive. The mounting block was used so that lateral accelerations could also be measured but these are not reported here. The accelerometer on the rail was located at the base of the rail web, the one on the tunnel invert was located directly under the rail close to the sleeper and the one on the tunnel wall was located at mid-height of the tunnel bore. All three were in the same cross-section. These measurements were recorded at a sample rate of $2.5 \mathrm{kHz}$ using a data logger that was installed in the tunnel during the overnight closure. A total of 85 train passages were recorded at these locations. The mean train speed was $11.5 \mathrm{~m} / \mathrm{s}(41.4$ $\mathrm{km} / \mathrm{h}$ ) with a standard deviation of $1.4 \mathrm{~m} / \mathrm{s}(5.04 \mathrm{~km} / \mathrm{h})$. Only a single train type (1972 Stock) was in operation. 
The above-ground measurements consisted of the vertical vibration on the surface of the pavement above the tunnel. These measurements were made early in the morning, between 05.30 and 07.00 when there was limited road traffic. The events corresponding to train passages in the tunnel under investigation were identified by synchronising the measurement equipment with that deployed in the tunnel. Two accelerometers were used that were each fixed to a small metal plate of mass $300 \mathrm{~g}$ by a threaded stud; the plates were placed on the pavement surface. For these measurements a sample rate of $750 \mathrm{~Hz}$ was used. Only 20 trains were measured here as a shorter measurement period had to be used than in the tunnel.

All the results shown below are expressed as averages over the train length and converted to $1 / 3$ octave band spectra by integration of the power spectral density over the corresponding frequency bands.

The rail roughness of both rails at the site was measured using a CAT trolley. The resulting average spectrum is shown as the solid black line in Figure 7. This covered the wavelength range up to $1.6 \mathrm{~m}$. Longer wavelengths are included in the model by using an extrapolated spectrum. Measurements of the wheel roughness were not available. The wheel roughness is not expected to be significant at long wavelengths but is likely to contribute to the excitation at shorter wavelengths. The total roughness at short wavelengths has also been determined indirectly from the rail vibration using a pass-by method, as described in Section 5.3 below. The total roughness spectra obtained from this indirect method is also shown in Figure 7, as the dashed line.

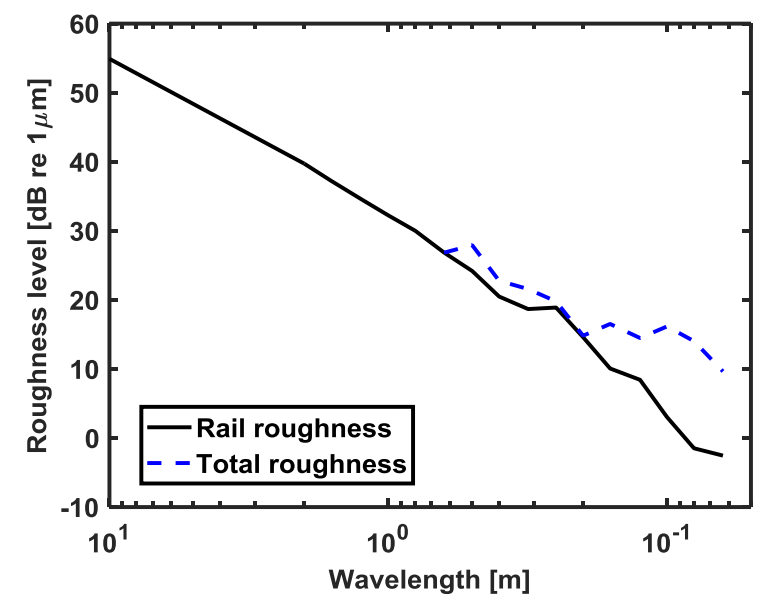

Figure 7 Directly measured rail roughness and indirectly measured total roughness in $1 / 3$ octave bands

Measurements were made of the track mobility and decay rate from which the rail pad stiffness and damping could be extracted using the method given in [40]. The rail pad 
stiffness was identified as $2.62 \times 10^{8} \mathrm{~N} / \mathrm{m}^{2}$ (per unit length of rail) and the damping loss factor as 0.12 .

\subsection{Model parameters}

The trains consisted of seven-coach units with a mixture of four powered vehicles and three trailer vehicles. In the calculations, to avoid the need for an excessively long ground model, this is simplified to a four-vehicle train consisting of two driving cars and two trailer cars. Although shorter than the actual seven-coach train, this is considered to be sufficient to give a reasonable estimate of the average level during a pass-by, as the train length, $64 \mathrm{~m}$ in the model, is large compared with the tunnel depth. Moreover, from simulations with trains of a single type of vehicle, the effect on the average spectrum of having half the vehicles powered compared with $4 / 7$ is only around $0.5 \mathrm{~dB}$.

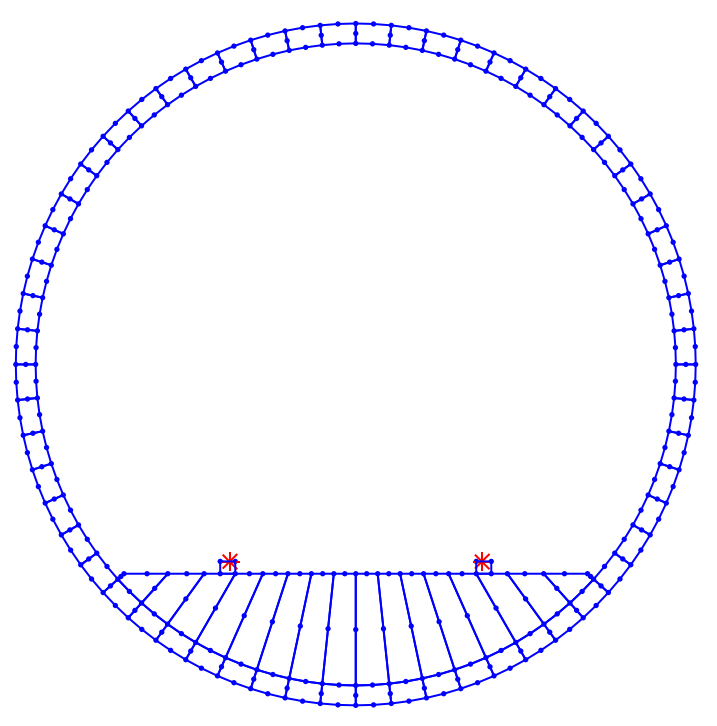

Figure 8 Cross-section of track-tunnel finite element model

The cross-section of the tunnel-track model is shown in Figure 8. The tunnel structure is modelled by 8-noded quadrilateral solid elements. For simplicity, the sleepers and the gravel filling are included as part of the concrete invert. The thickness of the invert is maintained even though the equivalent lining is thicker than the actual one. The two rails are modelled by beam elements with vertical bending stiffness of $4.86 \times 10^{6} \mathrm{Nm}^{2}$ and mass per unit length of $56 \mathrm{~kg} / \mathrm{m}$. The rail pads are modelled as an equivalent orthotropic material giving the same vertical stiffness per unit length $\left(2.62 \times 10^{8} \mathrm{~N} / \mathrm{m}^{2}\right)$ as the discrete pads in the track, see Section 5.1. The damping loss factor of the rail pads is 0.12 . 
The soil is included in the model by using boundary elements around the perimeter of the tunnel and along the ground surface. The ground surface is modelled to $30 \mathrm{~m}$ on the right hand side. The left hand side is truncated at $-10 \mathrm{~m}$ in order to limit the model size. The soil properties used in the ground model are based on London clay as given in Table 3 [31]. The corresponding shear wave speed is $220 \mathrm{~m} / \mathrm{s}$ and the longitudinal wave speed is 1571 $\mathrm{m} / \mathrm{s}$. The damping loss factor is 0.078 .

The wavenumber range chosen in the calculation is from -16 to $16 \mathrm{rad} / \mathrm{m}$, linearly spaced with 2048 wavenumbers giving a wavenumber resolution of $0.016 \mathrm{rad} / \mathrm{m}$. The frequency range of interest is up to $250 \mathrm{~Hz}$ which covers the expected range of groundborne vibration and noise. The frequencies calculated are logarithmically spaced with three frequencies per one-third octave band. The results are converted into one-third octave bands for presentation.

Table 3 Material properties

\begin{tabular}{|c|c|c|c|}
\hline & Young's Modulus & Poisson's ratio & Density \\
\hline Equivalent Isotropic Cylinder & $19.1 \mathrm{GPa}$ & 0.3 & $2104 \mathrm{~kg} / \mathrm{m}^{3}$ \\
\hline Invert (Concrete C50) & $50 \mathrm{GPa}$ & 0.3 & $2500 \mathrm{~kg} / \mathrm{m}^{3}$ \\
\hline Soil (London clay) & $0.286 \mathrm{GPa}$ & 0.49 & $1980 \mathrm{~kg} / \mathrm{m}^{3}$ \\
\hline
\end{tabular}

\subsection{Quasi-static and dynamic components}

The quasi-static and dynamic components of vibration on the rail and tunnel structure predicted using the model introduced above are compared in Figure 9. The quasistatic part is predicted using the method described in Section 4.4 while the dynamic part is given by the 2.5D WANDS model coupled with the vehicle model.

It can be seen that the quasi-static component of vibration dominates at low frequencies, up to $16 \mathrm{~Hz}$ on the rail, but decays rapidly with increasing frequency especially for positions further from the rail. The fluctuations at low frequencies (below $8 \mathrm{~Hz}$ ) in both quasi-static and dynamic responses occur due to the axle spacing [2]. At high frequencies, the total vibration on the track and the tunnel structure is dominated by the dynamic component. In these figures the dynamic component is predicted using only the rail roughness.

For the vibration on the ground surface (not shown here), the quasi-static vibration is much smaller than the dynamic component for all frequencies considered. 

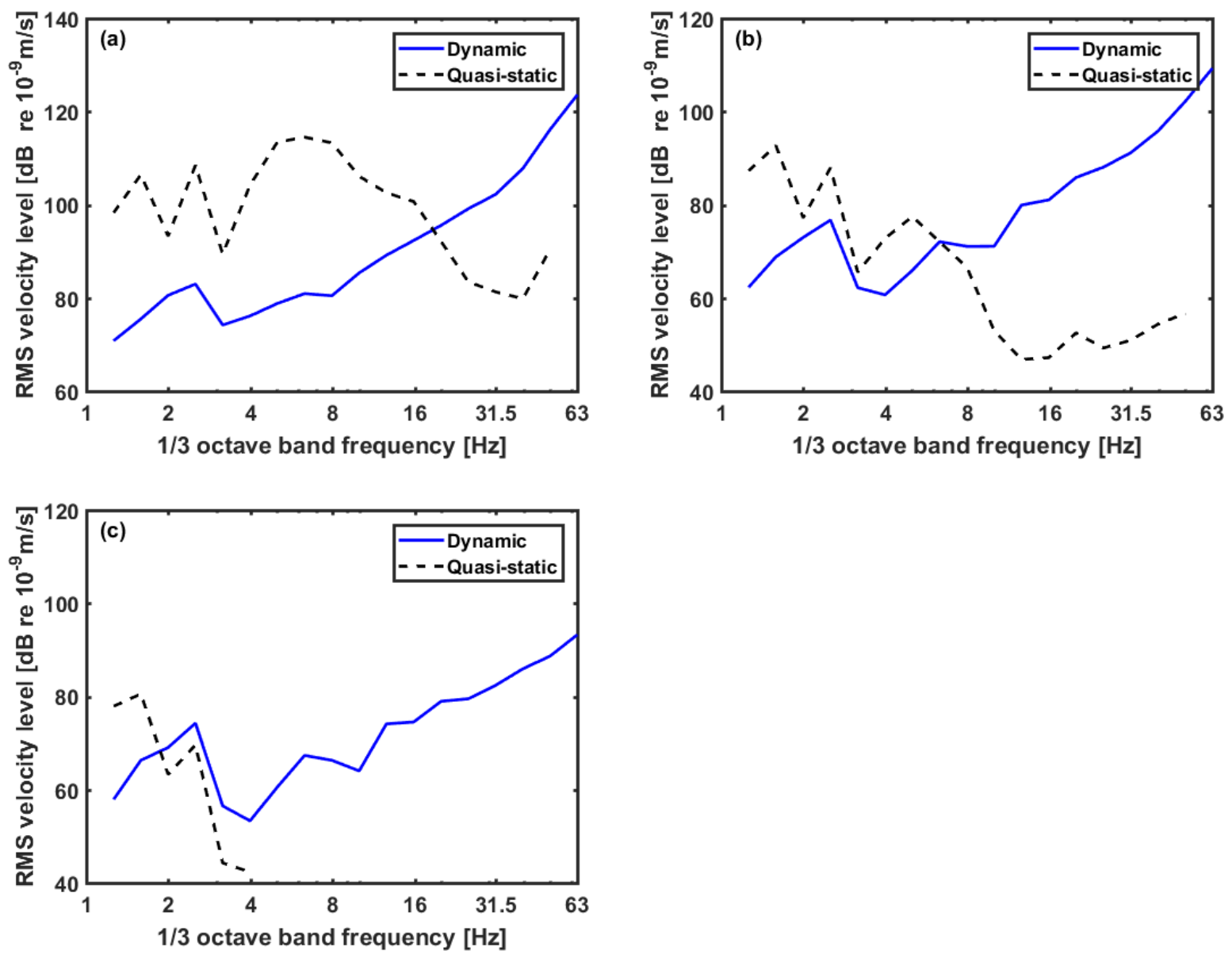

Figure 9 Quasi-static and dynamic components of vibration on the track and tunnel: (a) on the rails; (b) on the tunnel invert; (c) on the tunnel wall.

\subsection{Vibration on the rail and tunnel structure}

Figure 10 compares the total predicted level (spectral sum of the quasi-static and dynamic components) of rail vibration, obtained using the coupled 2.5D FE-BE model, with the measurement results. The dash-dot line shows the total vibration level predicted using only the directly measured rail roughness. Above $50 \mathrm{~Hz}$, the predictions are about 10 $\mathrm{dB}$ lower than the measurements. This is believed to be due to the omission of wheel unevenness, which was not available. To rectify this, the measured rail vibration can be used to estimate the total roughness indirectly [41]. This can only be done for frequencies where the dynamic component dominates the rail vibration, which in the present case corresponds to frequencies above $20 \mathrm{~Hz}$, wavelengths less than $0.25 \mathrm{~m}$, see Figure 9(a).

The dashed line in Figure 10 shows the prediction obtained using the estimated total roughness of the rail and wheel. This matches the measurement exactly at frequencies 
above $25 \mathrm{~Hz}$, as this rail vibration measurement is used to determine the total roughness. The peak in the response at around $80 \mathrm{~Hz}$ is due to the resonance frequency of the coupled vehicle/track system, which was seen as the crossing point of the wheel and rail mobilities in Figure 5.

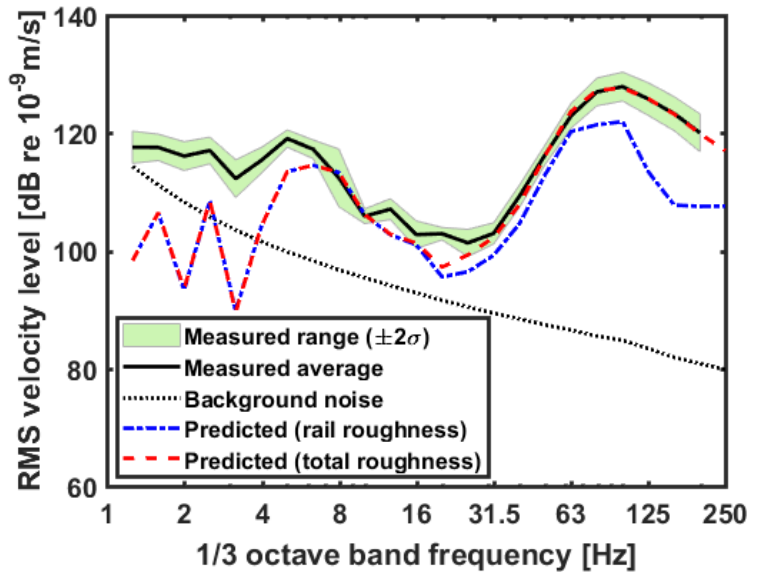

Figure 10 Comparison of measured and predicted rail vibration

Using the total roughness obtained from the rail vibration, the predictions for the vibration on the tunnel invert and the side wall show a good agreement with the measurements, as presented in Figure 11. The fluctuations below $10 \mathrm{~Hz}$ (as in Figure 9) are not observed in the measurements, probably due to the influence of background noise in the measurements.
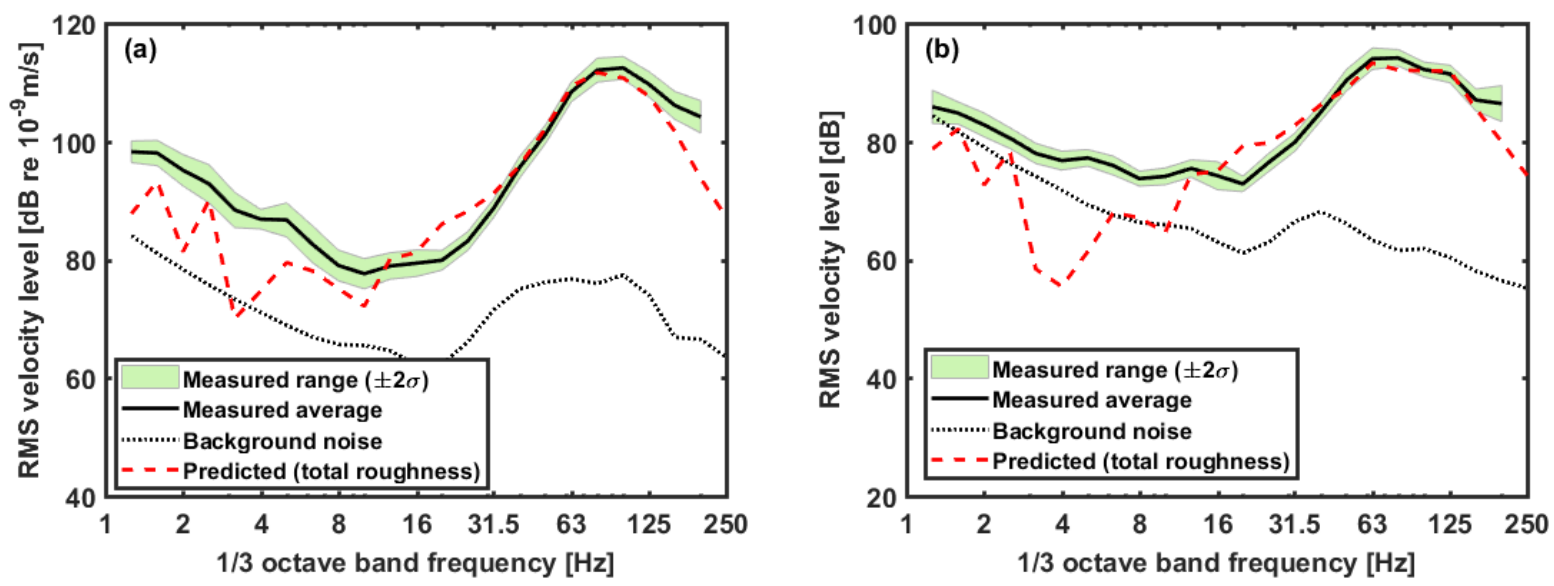

Figure 11 Comparison of measured and predicted vibration: (a) on tunnel invert; (b) on the tunnel wall.

\subsection{Vibration on the ground surface}

Figure 12 shows a comparison of the vibration on the ground surface above the tunnel predicted using the model with that obtained from the measurements. The 
background noise is also shown for comparison. Here, the quasi-static component of vibration is negligible and the ground vibration shown in the prediction is entirely due to the dynamic component.

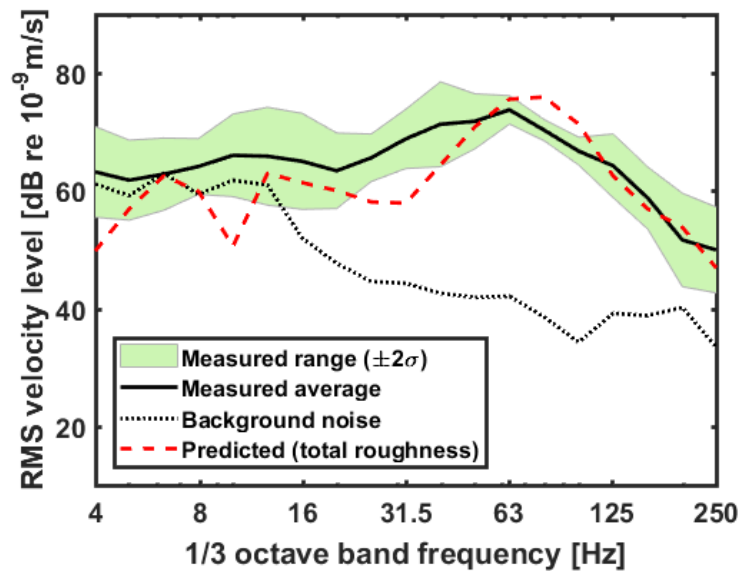

Figure 12 Comparison of measured and predicted vibration at ground surface

It can be seen that the predictions agree broadly with the measurements. The peaks appearing in the predictions at low frequencies are again due to the axle spacing; these cannot be detected in the measurements at the ground surface due to the influence of background noise. The peak in the response at around $80 \mathrm{~Hz}(63 \mathrm{~Hz}$ in the measurements), which is due to the resonance frequency of the train/track system, is present in both the measurements and predictions but has a higher level in the predictions.

Two additional calculations have been performed with modified ground properties. In the first, a stiffer upper layer was added in the top $5 \mathrm{~m}$ of the ground with $25 \%$ higher wave speeds. In the second, a heavier layer with $14 \%$ higher density and depth $0.5 \mathrm{~m}$ was added to represent the paving. In both cases the differences in ground vibration were negligible.

\section{Conclusions}

A prediction procedure has been presented for the vibration induced by a train running in a tunnel, based on the 2.5D FE-BE methodology. Results are also presented from a series of measurements that are used for validation of the model.

In the particular case considered, the tunnel structure consists of bolted cast-iron segments, the flanges of which act as periodic stiffeners. To represent this in the $2.5 \mathrm{D}$ approach an equivalent continuous structure is required. The properties of an equivalent isotropic cylinder are determined by comparing its dispersion relation with that obtained by 
a finite element model of the stiffened cylinder. The overall mass is conserved as well as the wavenumbers of the main bending and extensional waves and the cut-on frequencies of higher order modes.

Both the quasi-static and dynamic components of vibration are evaluated. The dynamic component of vibration, which is excited by the unevenness of the rail and wheel, is obtained by coupling the 2.5D FE-BE tunnel-ground model with a train consisting of 10 DOF multi-body vehicles. Using the same tunnel-ground model, the quasi-static component caused by the moving axle loads can be obtained through linear superposition of the deflection due to the passage of a single moving load. In the spectra of the total vibration, the quasi-static component is only found to be significant at low frequencies and only on the rail and the tunnel structure. Fluctuations are observed in the spectrum due to the axle spacing at frequencies below $10 \mathrm{~Hz}$.

The vibration levels on the rail, tunnel and ground surface are compared with the measurements. Using the measured rail roughness the vibration is under-predicted at frequencies above $50 \mathrm{~Hz}$. This is believed to be due to the omission of wheel roughness. The total roughness is therefore estimated from the rail vibration at frequencies above 25 $\mathrm{Hz}$, where the dynamic component is dominant. Using this roughness spectrum, the predicted levels show a good agreement with the measurement for the tunnel. At the ground surface the agreement is reasonable although the main peak at $63-80 \mathrm{~Hz}$ is over-predicted.

\section{Acknowledgements}

The first author was supported by a scholarship from the China Scholarship Council. The experimental work has been supported by the EPSRC research grant EP/K006002/1; EP/K005847/2, "MOTIV: Modelling of Train Induced Vibration". The authors are grateful to London Underground for their assistance. All data published in this paper are openly available from the University of Southampton repository at http://doi.org/10.5258/SOTON/D0416.

\section{References}

[1] D.J. Thompson, Railway Noise and Vibration: Mechanisms, Modelling and Means of Control. Elsevier: Oxford, 2009.

[2] G. Lombaert, G. Degrande, S. François, D.J. Thompson, Ground-borne vibration due to railway traffic: A review of excitation mechanisms, prediction methods and mitigation 
measures. In Nielsen, J.C.O., et al. (eds.) Noise and Vibration Mitigation for Rail Transportation Systems. Notes on Numerical Fluid Mechanics \& Multidisciplinary Design 126 (2015) 603-610.

[3] C. Weber, P. Karantonis, Rail ground-borne noise and vibration prediction uncertainties. Proceedings of 12th International Workshop on Railway Noise (IWRN), Terrigal, Australia, September 2016.

[4] J.P. Talbot, Base-isolated buildings: towards performance-based design. Proceedings of the Institution of Civil Engineers-Structures and Buildings 169 (2016) 574-582.

[5] Transit noise and vibration impact assessment. Office of Planning and Environment, Federal Transit Administration (FTA), FTA-VA-90-1003-06; 2006

[6] H. Verbraken, G. Lombaert, G. Degrande, Verification of an empirical prediction method for railway induced vibrations by means of numerical simulations. Journal of Sound and Vibration, 330 (2011) 1692-1703.

[7] R.A. Hood, R.J. Greer, M. Breslin, P.R. Williams, The calculation and assessment of ground-borne noise and perceptible vibration from trains in tunnels. Journal of Sound and Vibration, 193 (1996) 215-225.

[8] C. Madshus, C. Bessason, L. Harvik, Prediction model for low frequency vibration from high speed railways on soft ground. Journal of Sound and Vibration, 193 (1996), 195203.

[9] D.P. Connolly, P.A. Costa, G. Kouroussis, P. Galvin, P.K. Woodward, O. Laghrouche, Large scale international testing of railway ground vibrations across Europe. Soil Dynamics and Earthquake Engineering, 71 (2015) 1-12.

[10] J.A. Forrest, H.E.M. Hunt, A three-dimensional tunnel model for calculation of traininduced ground vibration. Journal of Sound and Vibration, 294(4) 678-705, 2006.

[11] M.F.M. Hussein, H.E.M. Hunt, L. Rikse, S. Gupta, G. Degrande, J.P. Talbot, S. François, M. Schevenels, Using the PiP model for fast calculation of vibration from a railway tunnel in a multi-layered half-space. In Schulte-Werning, B., et al. (eds.) Noise and Vibration Mitigation for Rail Transportation Systems. Notes on Numerical Fluid Mechanics \& Multidisciplinary Design 99 (2008) 136-142.

[12] Y.B. Yang, H.H. Hung, D.W. Chang, Train-induced wave propagation in layered soils using finite/infinite element simulation. Soil Dynamics and Earthquake Engineering 23 (2003) 263-278. 
[13] G. Kouroussis, O. Verlinden, C. Conti, Ground propagation of vibrations from railway vehicles using a finite/infinite-element model of the soil. Proceedings of the Institution of Mechanical Engineers, Part F: Journal of the Rail and Rapid Transit, 223 (2009), 405413.

[14] D.P. Connolly, A. Giannopoulos, M.C. Forde, Numerical modelling of ground borne vibrations from high speed rail lines on embankments. Soil Dynamics and Earthquake Engineering, 46 (2013) 13-19.

[15] P. Lopes, P.A. Costa, M. Ferraz, R. Calçada, A.S. Cardoso, Numerical modelling of vibrations induced by railway traffic in tunnels: From the source to the nearby buildings. Soil Dynamics and Earthquake Engineering, 61 (2014) 269-285.

[16] C.J.C Jones, D.J. Thompson, M. Petyt, A model for ground vibration from railway tunnels. Proceedings of the Institution of Civil Engineers-Transport 153 (2002) 121-129.

[17] L. Andersen, C.J.C. Jones, Coupled boundary and finite element analysis of vibration from railway tunnels - a comparison of two-and three-dimensional models. Journal of Sound and Vibration, 293 (2006) 611-625.

[18] Y.B. Yang, H. H. Hung, A 2.5D finite/infinite element approach for modelling viscoelastic bodies subjected to moving loads, Int. J. for Numer. Meth. in Eng., 51 (2001) $1317-1336$.

[19] P. Jean, C. Guigou, M. Villot, A 2.5D BEM model for ground-structure interaction, Building Acoustics 11 (2004), 157-173.

[20] M. Villot, P. Ropars, P. Jean, E. Bongini, F. Poisson, Modelling the influence of structural modifications on the response of a building to railway vibration. Noise Control Engineering Journal 59 (2011), 641-651.

[21] X. Sheng, C.J.C. Jones, D.J. Thompson, Modelling ground vibration from railways using wavenumber finite- and boundary-element methods. Proceedings of the Royal Society A, 461 (2005) 2043-2070.

[22] G. Lombaert, G. Degrande, Ground-borne vibration due to static and dynamic axle loads of InterCity and high-speed trains. Journal of Sound and Vibration, 319 (2009) 10361066.

[23] P. Alves Costa, R. Calçada, A.S. Cardoso, Track-ground vibrations induced by railway traffic: In-situ measurements and validation of a 2.5D FEM-BEM model. Soil Dynamics and Earthquake Engineering, 32 (2012) 111-128. 
[24] A. Dijckmans, P. Coulier, J. Jiang, M.G.R. Toward, D.J. Thompson, G. Degrande, G. Lombaert, Mitigation of railway induced ground vibration by heavy masses next to the track. Soil Dynamics and Earthquake Engineering, 75 (2015) 158-170.

[25] D.J. Thompson, J. Jiang, M.G.R. Toward, M.F.M. Hussein, E. Ntotsios, A. Dijckmans, P. Coulier, G. Degrande, G. Lombaert, Reducing railway-induced ground-borne vibration by using open trenches and soft-filled barriers. Soil Dynamics and Earthquake Engineering 88 (2016) 45-59.

[26] Y.B. Yang, H.H. Hung, Soil vibrations caused by underground moving trains. Journal of Geotechnical and Geoenvironmental Engineering 134 (2008) 1633-1644.

[27] Y.B. Yang, X. Liang, H.H. Hung, Y. Wu, Comparative study of 2D and 2.5D responses of long underground tunnels to moving train loads. Soil Dynamics and Earthquake Engineering, 97 (2017) 86-100.

[28] H.H. Hung, G. H. Chen, and Y. B. Yang, Effect of railway roughness on soil vibrations due to moving trains by 2.5D finite/infinite element approach, Eng. Struct., 57 (2013) 254-266.

[29] P. Amado-Mendes, P.A. Costa, L.M. Godinho, P. Lopes, 2.5 D MFS-FEM model for the prediction of vibrations due to underground railway traffic. Engineering Structures, 104 (2015) 141-154.

[30] A. Yaseri, M.H. Bazyar, and S. Javady. 2.5 D coupled FEM-SBFEM analysis of ground vibrations induced by train movement. Soil Dynamics and Earthquake Engineering 104 (2018) 307-318.

[31] G. Degrande, D. Clouteau, R. Othman, M. Arnst, H. Chebli, R. Klein, P. Chatterjee, B. Janssens, A numerical model for ground-borne vibrations from underground railway traffic based on a periodic finite element-boundary element formulation. Journal of Sound and Vibration, 293 (2006) 645-666.

[32] C.M. Nilsson, C.J.C. Jones, Theory manual for WANDS 2.1. ISVR Technical Memorandum 975, University of Southampton, 2007.

[33] M. Petyt, Introduction to finite element vibration analysis. Cambridge University Press, $2^{\text {nd }}$ edition, 2010.

[34] X. Sheng, C.J.C. Jones, D.J. Thompson, Computer programs for the discrete wavenumber finite/boundary element model for ground vibration and noise from 
railway tunnels. ISVR Technical Memorandum 915, University of Southampton, 2003.

[35] C.M. Nilsson, C.J.C. Jones, D.J. Thompson, J. Ryue, A waveguide finite element and boundary element approach to calculating the sound radiated by railway and tram rails. Journal of Sound and Vibration 321 (2009) 813-836.

[36] L.H. Donnell, A discussion of thin shell theory. Proceedings of the Fifth International Congress on Applied Mechanics, 1938.

[37] W. Sun, J. Zhou, D.J. Thompson, D. Gong, Vertical random vibration analysis of vehicle-track coupled system using Green's function method. Vehicle System Dynamics, 52(2014), 362-389.

[38] E. Ntotsios, D.J. Thompson, and M. Hussein. The effect of track load correlation on ground-borne vibration from railways. Journal of Sound and Vibration 402 (2017): 142-163.

[39] K.A. Kuo, H.E.M. Hunt and M.F.M. Hussein, The effect of a twin tunnel on the propagation of ground-borne vibration from an underground railway. Journal of Sound and Vibration, 330(2011), 6203-6222.

[40] G. Squicciarini, D.J. Thompson, M.G. Toward, and R.A Cottrell, The effect of temperature on railway rolling noise. Proceedings of the Institution of Mechanical Engineers, Part F: Journal of Rail and Rapid Transit. 230.8 (2016) 1777-1789.

[41] M.H.A. Janssens, M.G. Dittrich, F.G. de Beer, C.J.C. Jones, Railway noise measurement method for pass-by noise, total effective roughness, transfer functions and track spatial decay, Journal of Sound and Vibration 293 (2006), 1007-1028. 